\title{
The optimal resolution of garment 3D model based on geometric deviation and curvature analysis
}

\author{
XIAO Wei-min ${ }^{1}$, ZHONG Yue-qii ${ }^{1,2}$ and WU Ge ${ }^{1}$ \\ ${ }^{1}$ College of Textiles, Donghua University, Shanghai 201620, China \\ ${ }^{2}$ Key Lab of Textile Science and Technology, Ministry of Education, P. R. China \\ *zhyq@dhu.edu.cn(Corresponding author)
}

Keywords: 3D garment model; resolution; similarity; curvature

\begin{abstract}
The data volume of 3D garment model is closed related to its visual quality. Considering the limitation of model data in real-time rendering, it is necessary to study the relationship between them and find the optimal resolution which meets the demands of the real-time rendering. Simplified 3D garment models with different resolutions were obtained from the origin model. Similarity and curvature distribution were used to characterize their visual effects. The relationship between model data and their visual effects were analyzed. Meredith method was used to calculate the optimal resolution of 3D garment model. The results show that the optimal resolution of 3D garment model can be obtained effectively by Meredith method. A 90\% reduction in the data volume of 3D garment model will not have a significant influence on its visual effect.
\end{abstract}

\section{Introduction}

The 3D garment model made by 3D scanner or CAD software has been applied in many fields, such as virtual fitting and online exhibition [1][2]. Since the data volume of 3D garment is generally larger than the need of online browsing, it is necessary to study the relationship between the data volume and the visual effect of 3D garment and find the optimal resolution that can show its detail with minimum data volume.

The visual effect of 3D model can be evaluated with the paramter such as similarity, curvature distribution and roughness[3]. P. Cignoni et al[4] proposed Hausdorff distance to character the similarity between original model and simplified model. D.Luebke et al[5] utilized the average, the RMS , and combinations of Hausdorff distances to represent the similarity, which is more rational than before. Wu et al[6] adopted the biggest dihedral angle to replace the curvature of 3D model, their method could increased the efficiency of simplification and the detail of 3D model can be well reserved. Roughness was utilized to evaluate the visual effect of simplified 3D model, and their test result was similar with that of subjective assessment in papers[7,8].

However, most of the research above adopted single parameter. It[9] had been pointed out that the visual effect could not be evaluated correctly with single parameter compared to combined parameter. Among the common parameters, similarity and curvature distribution are the simplest and the most important two. The former could be utilized to reflect the whole deformation of 3D model[10]. The latter could be employed to characterize the micro deformation, which is easy to observed by naked eye but difficult to judge by similarity. Therefore, the combination of these two parameters would contribute a better assessment to the visual effect.

In this paper, triangle models were chosen for simplification. A series of simplified 3D models were obtained with different resolutions. Similarity and curvature distribution were selected to assess their visual effect. The relationship between data volume and visual effect was quantitative analyzed, and the optimal resolution for 3D garment model is calculated.

\section{Experiment}

1.1 Construction of 3D garment models

(1) Garment sample selection 
The common garments can be divided into 5 types: pants, shirt, skirt and knitwear. To guarantee the representation of the sample, 10 samples were selected from these 5 types: jeans, shirt, jacket, business suit, coat, braces skirt, longuette, T-shirt, fleece and sweater.

(2) Approach of model construction

This paper constructed models based on the Kinect and KinectFusion algorithm. Kinect was a 3D camera developed by Microsoft, which is capable of simultaneously capturing a color image and depth image information. KinectFusion algorithm positioning and integration can be matched by the Kinect depth data collected to achieve a three-dimensional reconstruction of real-time and with high precision, which had been widely used virtual fitting, scene reconstruction and somatosensory games and other fields.

\subsection{Simplification of original models}

We simplified the original clothing models with simplification algorithm using quadric error metrics and based on edge contraction. The basic deleted element was edge in edge contraction, and after several iterative edge contractions, we got the resolution we want. The specific process shown in Fig.1, vertex $v_{1}$ and $v_{2}$ contracted to a single vertex $\overline{\mathrm{V}}$, the two shaded triangles that shared edge $v_{1} v_{2}$ were deleted, and thereby simplified operation was finished.
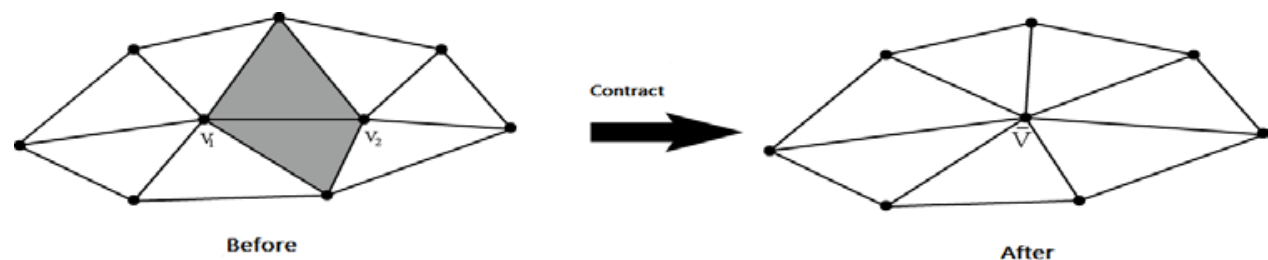

Fig.1Diagram of QEM simplification

Our simplification algorithm is built around pair contractions and error quadrics. The current implementation represents models using $n$ adjacency graph structure: vertices, edges, and faces are all explicitly represented and linked together. To track the set of valid pairs, each vertex maintains a list of the pairs of which it is a member. The algorithm itself can be quickly summarized as follows:

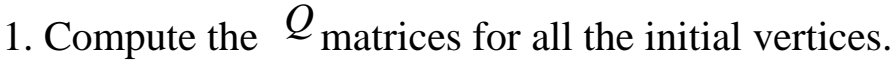

2. Select all valid pairs.

3. Compute the optimal contraction target $\bar{v}$ for each valid pair $\left(\mathrm{v}_{1}, \mathrm{v}_{2}\right)$. The error $\overline{\mathrm{v}}^{T}\left(\mathrm{Q}_{1}+\mathrm{Q}_{2}\right) \overline{\mathrm{v}}$ of this target vertex becomes the cost of contracting that pair.

4. Place all the pairs in a heap keyed on cost with the minimum cost pair at the top.

5. Iteratively remove the pair $\left(\mathrm{v}_{1}, \mathrm{v}_{2}\right)$ of least cost from the heap, contract this pair, and update the costs of all valid pairs involving $\mathrm{v}_{1}$.

The method can maintain the contours and geometric characteristics of the original model, while the fast calculation speed is fast, and thus become a classic edge contraction simplification algorithm. Using the algorithm described above to simplify model of garments, simplified rates were $10 \%$, 20\%, 30\%, 40\%, 50\%, 60\%, 70\%, 80\%, 90\%, 92\%, 94\%, 96\%, 98\% , 99\%, $99.5 \%$.

\subsection{Computation of geometric deviations}

The human eye can't find the difference between them, when simplified rates are $10 \%-94 \%$. In order to evaluate the differences between models, we took geometric deviations as our indicator to quantify the differences between models.

The approximation error between two meshes may be defined, as follows, as the distance between corresponding sections of the meshes. Given a point $\mathrm{P}$ and a surface $\mathrm{S}$, we define the distance $e(\mathrm{p}, \mathrm{S})$ as:

$$
e(\mathrm{p}, \mathrm{S})=\min _{p^{\prime} \in S} d\left(\mathrm{p}, \mathrm{p}^{\prime}\right)
$$

Where $d()$ is the Euclidean distance between two points in $\mathrm{E}^{3}$. 
Given a set of uniformly sampled distances, we denote the mean distance $E_{\mathrm{m}}$ [3] between two surfaces as the surface integral of the distance divided by the area of $S_{1}$ :

$$
E_{m}\left(S_{1}, S_{2}\right)=\frac{1}{\left|S_{1}\right|} \int_{S_{2}} d\left(p_{i}, S_{2}\right) d s
$$

If the surface $S_{1}$ is oriental, we can extend the definition of distance between a point $\mathrm{p}$ of $\mathrm{S}_{1}$ and $S_{2}$.

In this paper, we regard $S_{1}$ in the above formula as the original model, the simplified model as $S_{2}$, calculate the average distance between simplified models and original models. Then can we get accurate differences between these models.

\subsection{Computation of curvature distribution}

In addition to geometric deviation, another important property of model is that curvature. Geometric shape reflects the overall look and feel of the model, however curvature reflects the local features of model, whether model is smooth or has sharp portion, we can see from curvature. Theoretically Gaussian curvature is mainly used to present curvature of model.

A surface can be approximated by an interpolating quadratic surface. The normal and curvature of the quadratic surface are approximations to the normal and curvature of the surface.

A quadratic surface that passes through the origin is:

$$
z=A_{10} x+A_{01} y+\frac{A_{20}}{2} x^{2}+A_{11} x y+\frac{A_{02}}{2} y^{2}
$$

With the surface equation, Gauss Curvature can be solved. To analyze the relationship between the curvature distribution and simplified rate of model, we divided curvature of vertices into [0, $0.25 \pi],(0.25 \pi, 0.5 \pi],(0.5 \pi, 0.75 \pi]$ and $(0.75 \pi, \pi] 4$ intervals. Big Interval value means big curvature value. The vertices in corresponding interval are denoted as Class A, Class B, Class C and Class $D$ vertices. Thus, the percentage of vertices can be used to represent curvature distribution of models.

\section{2 results and discussion}

\subsection{Data curve}

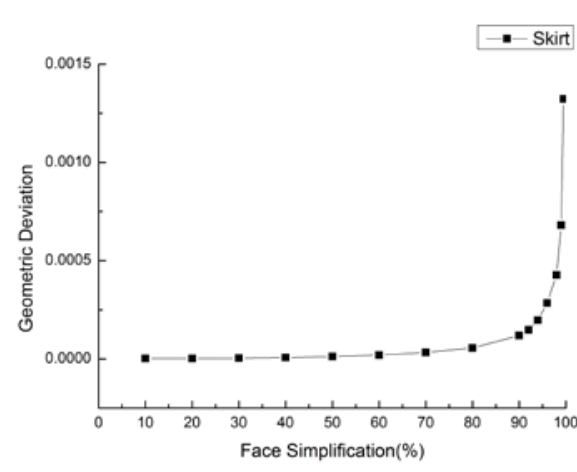

(a)

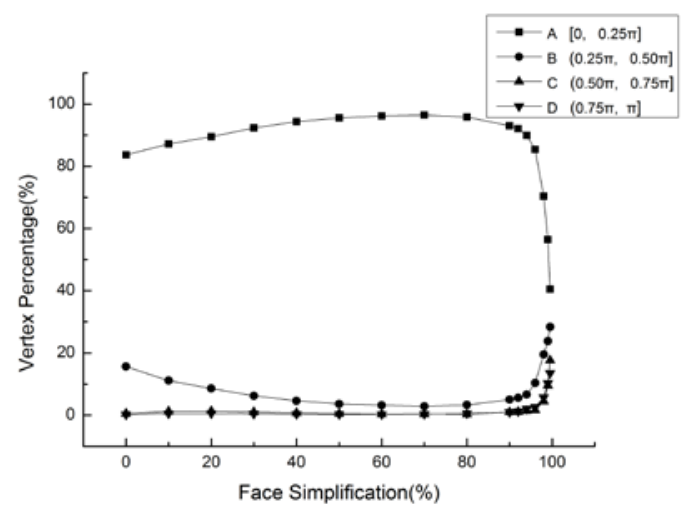

(b)

Fig.2 The interpolation curve of experimental data

After experiments above, we can obtain geometric deviation and curvature distribution between the original model and the simplification. Took shirt as an example, the data curve are shown in Fig.2 (a) showed the change of geometric deviation and (b) showed the change of curvature distribution. As can be seen from Fig. 2(a), when the simplified rate was less than $80 \%$, the geometric deviation between the original model and the simplified model changes was very little. When it was more than $80 \%$, geometric deviations began to increase significantly. As can be seen from Fig. 2(b), when simplified rates was less than 90\%, the percentage change in the four class of 
did not change much. And when simplified rates was greater than $90 \%$, the number of vertices Class A reduced quickly, while the other three class of vertices were increasing rapidly.

In addition, the percentage of A class vertices before dramatic changes was much higher than the other three categories vertices, accounting for over $80 \%$ of the model, which showed the majority of surface model described relatively smooth surface, is connected between the sharp facets less. This is consistent with the model the actual characteristics, also verified the feasibility of vertices adjacent triangles maximum curvature dihedral characterization methods.

\subsection{Calculation of optimal resolutions}

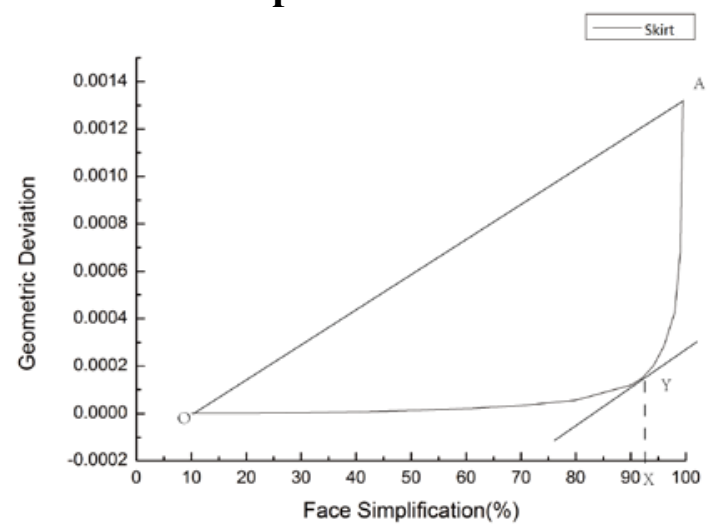

(a)

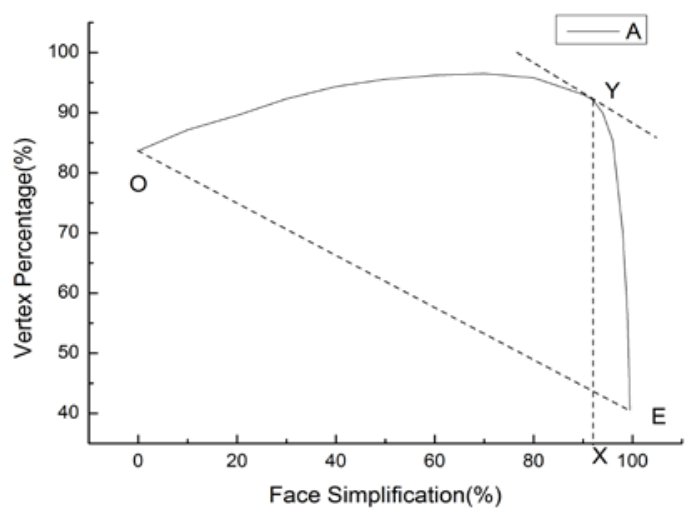

(b)

Fig.3 Diagram for finding the optimal resolution with Meredith method

Tab.1 Results of the optimal resolution with Meredith method

\begin{tabular}{ccccccccccc}
\hline $\begin{array}{c}\text { Clothing } \\
\text { styles }\end{array}$ & Jeans & Shirt & Jacket & Coat & Suit & Longuette & $\begin{array}{c}\text { braces } \\
\text { skirt }\end{array}$ & T-shirt & Fleeces & Sweater \\
\hline GD (\%) & 90 & 90 & 90 & 90 & 90 & 90 & 90 & 90 & 90 & 90 \\
CD (\%) & 90 & 90 & 90 & 90 & 90 & 90 & 90 & 90 & 90 & 90 \\
TN & 20196 & 18542 & 18963 & 21409 & 18807 & 18868 & 12795 & 15149 & 17899 & 20052 \\
\hline
\end{tabular}

In order to investigate the critical point of the curve from a small change to the dramatic changes, This article draws on the fiber tensile yield point curve method-Meredith method to determine the optimal simplified rate of the geometrical differences and the curvature of the curve This method can calculate the critical point easily and accurately, when the curve's characterization indicators change. For example in a shirt shown in Fig. 3, Fig. 3 (a) is the geometric deviation of the curvature curve, Fig. 3 (b) is a class A apex curvature's distribution curve. Connecting the initial point $\mathrm{O}$ and the end point E, making a line parallel to $\mathrm{OE}$ and tangent to the curve, the tangent point is the inflection point. The inflection point of the abscissa $\mathrm{X}$ is the optimal simplified rate. Because the percentage of Class A vertex is much higher than the other three types of vertices before the dramatic changes, In Fig. 3 (b) variation we using the changes of Class A's vertices to approximate assessments in the curvature changes of the model surface. We deal with the entire Three-dimensional clothing model in accordance with the method described above; we obtain their optimal simplified rate. According to the definition of resolution of 3D model, the optimal resolution is the number of facets at its optimal rate simplified model; the results are shown in Table1.

As can be seen from Table 1, (GD means optimal simplified rates based on geometric deviation, CD means optimal simplified rates based on curvature distribution, TN means optimal triangle numbers)the best simplified rates of selected 10 garments are $90 \%$ and were very stable, which based on curvature distribution and geometric deviations. The average of best number of triangles of models is about 16000, which was far less than the triangle number of their original model, so we can greatly accelerate the speed of browsing model online. The reason why the simplified rate remained stable at best $90 \%$ is the core QEM simplification algorithm is simplified from the 
smallest error cost of vertices direction, and the direction depends on the quadratic error, so the algorithm itself implicitly converges to a maximum permissible error. In its role as simplify the process proceeds to a specific extent will exceed this threshold, which results in quick increases of differences between a simplified model and the original model. And this threshold is not affected by the topology of the model, and the best simplified rate was a concrete manifestation of threshold based on the geometric error and curvature distribution, so it is relatively stable.

\section{Conclusion}

In this paper, we simplified garment model based on QEM simplification algorithm to varied degrees, then proposed dual characterization of visual evaluation of model which were geometric deviation and curvature. The relationship between the degree of simplification and visual effects were quantified assessment and proposed using the Meredith method to calculate the best simplified rate. The experimental results showed that: (1)We can calculate the optimal simplified rates stably and effectively in Meredith method, and then find their corresponding optimal resolutions (2) For majority of garment model, simplify its $90 \%$ of data volume does not significantly affect its visual effects. Browsing a simplified model of clothing can not only speed up browsing speed, but also can save computing resources. The work of this paper provided a theoretical basis for applications of online display and browsing.

\section{Acknowledgements}

This work was financially supported by the Shanghai Natural Science Foundation (14ZR1401100).

\section{References}

[1] A. Bulbul, T. Capin and G. Lavoué. Signal Proc. Mag. IEEE, Vol. 6 (2011), p. 80

[2] J. R. Cooperstock. Signal Proc. Mag. IEEE, Vol. 28 (2011), p.77

[3] L. Daly and D. Brutzman, Signal Proc. Mag. IEEE, Vol. 24 (2007), p.130

[4] P. Cignoni, C. Rocchini, and R. Scopigno. Computer Graphics Forum. Vol.17 (1998), p.167

[5] D. P. Luebke: Level of Detail for 3D Graphics (MKO Publications, USA 2003).

[6] J.-H. Wu, S.-M. Hu, C.-L. Tai, and J.-G. Sun, "An effective feature-preserving mesh simplification scheme based on face constriction," in Computer Graphics and Applications, 2001. Proceedings. Ninth Pacific Conference on, 2001, p. 12.

[7]E. D. Gelasca, T. Ebrahimi, M. Corsini, and M. Barni, in Image Processing, 2005. ICIP 2005. IEEE International Conference on, 2005, p. I-241.

[8] M. Corsini, E. D. Gelasca, T. Ebrahimi, and M. Barni, IEEE Transactions on Multimedia, Vol. 9(2007) p. 247

[9] A. Bulbul, T. Capin, G. Lavoué, and M. Preda, Signal Processing Magazine, IEEE, vol. 28, pp. 80-90, 2011. Signal Proc. Mag. IEEE, Vol. 28 (2011), p. 80

[10] M. Roy, S. Foufou, and F. Truchetet, International Journal of Image and Graphics, Vol.4(2004), p. 127 